$$
\left(y_{2} y_{1}^{\prime}-y_{1} y_{2}^{\prime}\right)(x)=\int_{a}^{x} y_{2}(s) y_{1}(s)\left[F\left(y_{2}^{2}(s), s\right)-F\left(y_{1}^{2}, s\right)\right] d s .
$$

By hypothesis $\left(y_{2} y_{1}^{\prime}-y_{1} y_{2}^{\prime}\right)(b)=0$. The integrand in $(7)$ is positive, however, as long as $y_{2}(s)>y_{1}(s)$ and in particular on some interval $(a, \alpha)$ - because $\left(y_{2}-y_{1}\right)^{\prime}(a)>0$. In fact, $\alpha$ may be taken as $b$ because the same argument as in Lemma 1 shows that the graphs of $y_{2}(x)$ and $y_{1}(x)$ can not intersect on $(a, b)$. Thus the right side of $(7)$ does not tend to zero as $x \rightarrow b-$.I

PROOF OF THE THEOREM. The existence of at least one solution of $(1)+(C)$ has been proved by Nehari [1, Theorem IV]. By the preceding lemmas there is at most one such solution.

\title{
REFERENCE
}

1. Zeev Nehari, On a class of nonlinear second-order differential equations, Trans. Amer. Math. Soc. 95 (1960), 101-123.

Massachusetts Institute of Technology

\section{ON THE MEASURABILITY OF FUNCTIONS IN TWO VARIABLES}

\author{
MARK MAHOWALD ${ }^{1}$
}

Let $(X, \mu)$ and $(Y, \nu)$ be two compact spaces having regular Borel measures defined on them. By a measurable modification $\tilde{f}(x, y)$ of a function $f(x, y)$ we mean a function measurable in both variables together and for which $\tilde{f}(x \cdot)=f(x \cdot)$ almost everywhere $[\nu]$ for every $x$. The purpose of this note is to prove the following theorem.

THEOREM. If $Y$ is metric and if $f(x, y)$ has a measurable modification and $f(x \cdot)$ is continuous for almost all $x$, then $f(x, y)$ is measurable in both variables together.

This theorem was discovered in an effort to prove that the Nelson canonical version [2] is measurable if it has a measurable modification. The theorem would prove this result except for the restriction that $Y$ be metric.

Received by the editors December 30, 1960 and, in revised form, April 14, 1960.

${ }^{1}$ Research supported by the United States Air Force Office of Scientific Research of the Air Research and Development Command under Contract No. AF 49(638)-265. Reproduction in whole or part is permitted for any purpose of the United States Government. 
We now prove the theorem; without loss of generality we can assume that $f(x \cdot)$ is continuous for all $x$. Let $\tilde{f}(x, y)$ be a measurable modification. By Lusin's theorem [1], we can find a compact subset $C$ of $X \times Y$ on which $\tilde{f}(x, y)$ is continuous and whose measure is greater than $1 / 2$ the total measure of $X \times Y$. We can choose $C$ such that every nonempty relatively open subset has positive measure. Let $\left\{U_{n}\right\}$ be a countable basis for the topology of $Y$. Then in particular $\left(X \times U_{n}\right) \cap C$ is empty or has positive measure. In either case $\nu\left(\left(\left(X \times U_{n}\right) \cap C\right)_{x}\right)$ is a measurable function of $x$. Let $A_{n}$ $=\left\{x ; \nu\left(\left(\left(X \times U_{n}\right) \cap C\right)_{x}\right)\right\}=0$. Then $A_{n}$ is a measurable subset of $X$ and $B_{n}=\left(A_{n} \times Y\right) \cap\left(X \times U_{n}\right) \cap C$ has measure zero. Indeed $\left(B_{n}\right)_{x}=\varnothing$ if $x$ is not in $A_{n}$ and equals $\left(\left(X \times U_{n}\right) \cap C\right)_{x}$ if $x$ is in $A_{n}$. Hence $\nu\left(\left(B_{n}\right)_{x}\right)=0$ for all $x$ and, since $B_{n}$ is a measurable subset of $X \times Y$, Fubini's theorem implies that it has measure zero. Therefore, $\mathrm{U} B_{n}=D$ has measure zero.

Let $E=C-D$. First we show that $E_{x}$ is compact for each $x$. Let $N_{x}=\left\{n ; x \in A_{n}\right\}$. Then

$$
\begin{aligned}
E_{x} & =(C-D)_{x}=\left(\bigcap_{n=1}^{\infty}\left(C-B_{n}\right)\right)_{x} \\
& =\bigcap_{n \in N_{x}}\left(C_{x}-\left(B_{n}\right)_{x}\right) \\
& =\bigcap_{n \in N_{x}}\left(C_{x}-U_{n}\right) .
\end{aligned}
$$

This shows that $E_{x}$ is compact. If $E_{x}$ has positive measure then it is the support of $\nu$ restricted to it. To see this let $F_{x}$ be the support, then $C_{x}-F_{x}$ is relatively open and there exists $U \subset Y$ such that $C_{x}-F_{x}$ $=U \cap C_{x}$. Let $U_{n_{k}}$ be a sequence of sets of $\left\{U_{n}\right\}$ whose union is $U$. Then each $n_{k}$ is in $N_{x}$ and, therefore, $\bigcup_{n \in N_{x}} U_{n} \supset U$. Hence $E_{x} \subset F_{x}$; therefore $E_{x}=F_{x}$.

On $E, f(x \cdot)$ is continuous; therefore for each $x, f(x \cdot)$ is continuous on $E_{x}$ and equals $\tilde{f}(x \cdot)$ almost everywhere. Now if $\nu\left(E_{x}\right) \neq 0$ then Theorem 55.B of [1] implies they are equal everywhere. Hence $\tilde{f}(x, y)=f(x, y)$ for almost all $(x, y),[\mu \times \nu]$, in $E$. An inductive application of this procedure in the complement of $C$ will yield the theorem.

\section{REFERENCES}

1. P. R. Halmos, Measure theory, Van Nostrand, New York, 1950.

2. E. Nelson, Regular probability measures on function spaces, Ann. of Math. 69 (1959), 630-643.

Syracuse University 